\title{
The role of electrotherapy in reducing the pain of patients with knee osteoarthritis during the COVID-19 pandemic
}

\author{
ANTONESCU Elisabeta ${ }^{1,2}$, SILIȘTEANU Sînziana Călina ${ }^{3,4}$, TOTAN Maria ${ }^{1,5}$
}

\section{Corresponding author: SILIŞTEANU Sînziana Călina, E-mail: sinzi silisteanu@yahoo.com}

1. Lucian Blaga University of Sibiu, Faculty of Medicine, Sibiu, România 2. County Clinical Emergency Hospital, Sibiu, România 3. Railway Hospital Iasi - Specialty Ambulatory of Suceava 4. "Stefan cel Mare" University of Suceava FEFS-DSDU

5. Children's Hospital, Sibiu, Romania

\begin{abstract}
Introduction. Osteoarthritis is considered to be the most common form of arthritis and a leading disability cause worldwide, especially due to the painful symptom. The latter is a clinical marker in evaluating the limits of joint mobility and therefore, the pain reduction is a goal of the recovery treatment for patients with knee osteoarthritis. The purpose of this study was to show whether the pain phenomenon characteristic of knee osteoarthritis can be reduced by electrotherapy, even in the context of the COVID-19 pandemic. Material and method. The study lasted 5 months and included 171 patients diagnosed clinically and radiologically with knee osteoarthritis. The followed parameters were pain, physical dysfunction in daily activities, anxiety and quality of life. Results and discussions. The two groups of studied patients were homogeneous in terms of weight by gender and age groups. The evaluation of patients according to scales enabled the registration of statistically significant values, the value of $\mathrm{p}$ $<0.05$, which explains the validation of the working hypothesis. The feeling of pain is closely related to the level of anxiety. Conclusions. Analgesic electrotherapy significantly reduced the pain syndrome of the patients for whom it was used. It has been shown that the patients' anxiety can influence the pain phenomenon. Given the conditions caused by the Covid-19 pandemic, the anxiety of the patients who were in the outpatient department to receive recovery treatment was increased, but after the recovery treatment there was a decrease, so these patients' quality of life increased.
\end{abstract}

Keywords: pain, analgesic electrotherapy, knee osteoarthritis, recovery treatment,

\section{Introduction}

Osteoarthritis is considered to be the most frequent form of arthritis and a leading disability cause worldwide, especially due to the painful symptom that determines the patient to address the doctor. At first the pain has an intermittent character, then it becomes chronic and persistent, affecting the joint mobility and implicitly these patients' quality of life (1). The chronic pain is a risk factor in the increase in the physical disability.

The most vulnerable category of the population is represented by the elderly who have bone fragility, increased risk of falling down whereas the pain phenomenon is not always treated properly $(2,3)$. The knee osteoarthritis, due to pain, limitation of joint mobility and the affected age group, is considered a chronic condition that can cause significant functional disability, with medical and socio-economic costs (4).

The presence of the pain symptom as well as the degree of knee damage by osteoarthritis of the knee can influence both the mobility of patients diagnosed with this condition and the development of these patients' daily activities and quality of life (5).

When the physical activity of patients with knee osteoarthritis is affected, the functionality of other systems can be influenced, such as the respiratory system and the cardiovascular one (1).

The incidence of knee osteoarthritis is expected to increase significantly in the coming decade due to the aging of the world's population (6).
Murphy's study in 2008 (7) shows that the risk of developing a symptomatic form of knee osteoarthritis is estimated at approximately $40 \%$ (47\% in women and $40 \%$ in men ). This risk may reach even $60 \%$ in the obese persons, in comparison to $30 \%$ in the persons with normal weight or in the overweight persons. From an etiological point of view, the pain is multifactorial, involving both intra-joint and extra- joint risk factors (8). The pain due to knee osteoarthritis is explained by the fact that some anatomical structures (ligaments, tendons, joints) become more sensitive to painful stimuli, whereas the activity of nociceptors located in these structures is influenced (9). One of the technique used to explore osteoarticular functionality and changes is Raman spectroscopy (10).

A study of 2016 (11) shows that it is important to make a biopsychosocial clinical evaluation before using the recovery treatment. This is because pain involves several factors: somatic, behavioral, cognitive, social, emotional and motivational (12).

This study also tries to establish which is the dominant mechanism of pain (nociceptive, neuropathic sensitization or central neuropathy), but at the same time to evaluate the possible biopsychosocial factors involved in the pain phenomenon (11).

The pain symptom is a clinical marker in assessing the limitation of joint mobility and therefore pain reduction is 
one of the goals of recovery treatment for patients with knee osteoarthritis (13).

The study of Abdel-Aziem in 2018 (14) showed the effect of the physiotherapeutic program on knee osteoarthritis according to the intensity of the pain. This parameter, pain, is considered one of the factors responsible for the evolution of knee osteoarthritis.

Dworkin et al. in 2011 concluded that the pain and severity degree are correctly assessed by using the VAS scale and the WOMAC pain subscale (15).

It was found that people diagnosed with knee osteoarthritis had higher pain intensities in comparison to witness for the same level of pressure stimuli, but also for lower pain or pressure level (16).

Some research has shown the role and importance of spinal relief in the perception of pain, because at this level there are mechanisms that can modulate the nociceptive transmission.

The pain transmission can be influenced at this level by inflows coming from the periphery as well as from the superior nerve structures (17). In this context, electrotherapy in knee osteoarthritis is also used for analgesic purposes by blocking the conduction of the impulse through the sensitive nerve fibers.

The purpose of this study was to show whether the pain phenomenon characteristic of knee osteoarthritis can be diminished by electrotherapy, even in the context of the COVID-19 pandemic.

\section{Material and method}

The study was conducted over a period of 5 months, from April to August 2020, on outpatients and included a number of 171 patients diagnosed clinically and radiologically with knee osteoarthritis. They followed, in series, a number of 15 days of treatment, in a rhythm of 1 session per day. The used treatment was pharmacological, according to the guidelines (NSAIDs in general administration and in topical one, analgesics) (18) and non-pharmacological (weight loss, avoidance of rough terrain, prolonged orthostatism, electrotherapy and physiotherapy). The patients were evaluated in the beginning of the treatment and at its end, and also 6 weeks later, when they returned to control.

The criteria for the inclusion in the group were: patients with a clinical and radiological diagnosis of knee osteoarthritis over 3 months, over 40 years old, without comorbidities in decompensated stages, without neuromental disorders, patients who agreed to participate in the study.

The criteria for the exclusion from the group were: patients under the age of 40, who also had cardiac, respiratory, renal, digestive disorders in a decompensated stage, non-compliant, who did not agree to participate in the study.

The patients signed the consent for the applied recovery treatment, then the study was carried out by respecting the norms of ethics and deontology according to the legislation in force.

The followed parameters were pain, physical dysfunction in daily activities, anxiety and quality of life. For pain we applied the VAS scale (0- no pain, 10 - maximum pain) and also the WOMAC pain subscale. The static pain was assessed (while sitting, sleeping, orthostatism) and the dynamic pain (while walking, climbing / descending stairs) in which $0=$ no pain and $20=$ maximum pain.

For the physical dysfunction, the WOMAC subscale was considered (activities at home and outside), the value $0=$ optimal development of activities and $68=$ limitation of daily activities.

In assessing the patients' quality of life we used the QOL scale (16 means a quality of life influenced by nociceptive factors, the value $112=$ well-being, without pain or other symptoms). We considered it useful, in the situation created by the COVID - 19 pandemic, to apply the anxiety assessment scale strictly related to the disease, not the one determined by previous anxiety states. In this sense we used the State Trait Anxiety Inventory (S.T.A.I.) scale and namely the subscale STAI $-\mathrm{X} 1$ where the values vary from 20 (reduced anxiety) to 80 (increased anxiety).

For this purpose, for the recovery treatment the patients of the group were divided into 2 study groups, namely: the L1 group (83 patients) who received, in addition to the pharmacological treatment, low frequency currents (Transcutaneous electrical nerve stimulation -frequency of $15-100 \mathrm{~Hz}$, duration of $30-200 \mathrm{~ms}$, Intensity $10-40$ $\mathrm{mA}$, with the rapid installation of the analgesic effect), medium frequency (interferential -with analgesic effect by increasing the pain level and changing the pain perception, vasomotor, hyperemic and resorptive) and ultrasound (analgesic effect by inhibiting the transmission of nociceptive information, antiinflammatory due to vasomotor and metabolic action, but also muscle relaxant - frequency $1 \mathrm{MHz}$,intensity of 0.5 $0.7 \mathrm{~W} / \mathrm{cm}^{2}$, duration of 5 minutes, pulse application regime to eliminate the thermal effect (19).

The L2 group (88 patients) who received only pharmacological treatment (they showed increased anxiety in the context of the pandemic and did not want to participate in electrotherapy sessions).

However, the patients of the L2 group accepted to participate in individual physiotherapy sessions, as well as the patients in the L1 group.

The kinetotherapy program lasted for 30 minutes a session, it took place daily and included passive mobilizations, active mobilizations and active mobilizations with resistance, but also coordination and balance exercises, useful in recovering posture and gait.

The L1 group included 83 patients, of which 41 (49.39\%) were female and $42(50.61 \%)$ were male, whereas the L2 
study group included 88 patients, of which 7 (53.41\%) were female and 41 ( $46.59 \%)$ male.

Table no. 1. Distribution of patients according to the study groups and age groups

\begin{tabular}{|c|c|c|c|c|}
\hline \multirow[t]{2}{*}{ Characteristics } & \multicolumn{2}{|c|}{ LOT LI } & \multicolumn{2}{|c|}{ LOT L2 } \\
\hline & $\mathbf{n}$ & $\%$ & $\mathbf{n}$ & $\%$ \\
\hline Female/Male gender & $41 / 42$ & $49.39 / 50.6$ & $47 / 41$ & $53.41 / 46.59$ \\
\hline Age (Median, SD) & \multicolumn{2}{|c|}{$62 \pm 12.69$} & \multicolumn{2}{|c|}{$60.5 \pm 12.91$} \\
\hline 40-49 years & 19 & \begin{tabular}{|l}
22.89 \\
\end{tabular} & 21 & 23.86 \\
\hline 50-59 years & 18 & 21.69 & 21 & 23.86 \\
\hline $60-69$ years & 21 & 25.31 & 16 & 18.18 \\
\hline $70-79$ years & 17 & 20.47 & 23 & 26.14 \\
\hline$>80$ years & 8 & 9.64 & 7 & 7.96 \\
\hline
\end{tabular}

The demographic data in the study were: patients' age, gender, living environment and body mass index. The patients' average age was $62 \pm 12.69$ in the L1 group (min. 40, max. 82) and $60.5 \pm 12.91$ in the L2 group (min. 40, max. 84).

Table no. 2. Distribution of patients according to body mass index

\begin{tabular}{|c|c|c|c|c|}
\hline \multirow[t]{2}{*}{ Characteristics } & \multicolumn{2}{|c|}{ LOT LI } & \multicolumn{2}{|c|}{ LOT L2 } \\
\hline & $\mathbf{n}$ & $\%$ & $\mathbf{n}$ & $\%$ \\
\hline BMI $\left(\mathrm{Kg} / \mathbf{m}^{2}\right)$ & \multicolumn{2}{|c|}{$29.39 \pm 4.29$} & \multicolumn{2}{|c|}{$29.41 \pm 4.57$} \\
\hline Normal weight (19-24.99 & 13 & 15.66 & 14 & 15.91 \\
\hline Oveweight (25-29.99) & 38 & 45.78 & 39 & 44.32 \\
\hline Obesity $(>\mathbf{3 0})$ & 33 & 38.55 & 35 & 39.77 \\
\hline
\end{tabular}

\section{Statistical analysis}

The data obtained from the initial, final and control evaluations were statistically processed by using Microsoft Excel 10. The median, the standard deviation, and the t-student test were calculated to test the working hypothesis. The chosen level of statistical significance was $5 \%$, ie. its value should be $\mathrm{p}<0.05$.

\section{Results}

The pain evaluated by the VAS scale showed statistically significant results in both groups, registering in L1 group a decrease by $42.86 \%$ at the end of the treatment and $50 \%$ during the control, in comparison to L2 group in which the pain decreased by $28.57 \%$ at the end of the treatment and by $40 \%$ during the control. The pain phenomenon assessed with the WOMAC subscale registered a decrease by $28.57 \%$ in the $\mathrm{L} 1$ group at the end of the treatment and of 50\% during the control, whereas in the L2 group the reduction of pain was by $21.43 \%$ at the end of the treatment and by $45.46 \%$ during the control.

The body mass index decreased in the L1 group by $2.44 \%$ at the end of the treatment and by 2.55 during the control, whereas in the L2 group it decreased by $1.59 \%$ at the end of the treatment and by $2.69 \%$ during the control. Also, the daily activities improved in the L1 group by $36.17 \%$ at the end of the treatment and by $50 \%$ during the control, whereas in the L2 group the improvement was by $29.41 \%$ at the end of the treatment and by $50 \%$ during the control. Anxiety decreased in the L2 group by $40.47 \%$ at the end of the treatment and by $32.25 \%$ during the control, whereas in the L2 group, anxiety decreased by
$36.84 \%$ at the end of the treatment and by $29.63 \%$ during the control.

Table no. 3. Evolution of the parameters in the two groups

\begin{tabular}{|l|c|c|c|c|c|c|}
\hline \multirow{2}{*}{ SCALES } & \multicolumn{3}{|c|}{ L1 GROUP } & \multicolumn{3}{c|}{ L2 GROUP } \\
\cline { 2 - 7 } & $\mathrm{Tl}$ & $\mathrm{T} 2$ & $\mathrm{~T} 3$ & $\mathrm{Tl}$ & $\mathrm{T} 2$ & $\mathrm{~T} 3$ \\
\hline BMII & $29.39 \pm 4.29$ & $28.67 \pm 4.3227 .94 \pm 4.29$ & $29.41 \pm 4.57$ & $28.94 \pm 4,55$ & $28.16 \pm 4.46$ \\
\hline VAS & $7 \pm 1.13$ & $4 \pm 0.46$ & $2 \pm 0.51$ & $7 \pm 1.89$ & $5 \pm 1.49$ & $3 \pm 1.19$ \\
\hline WOMAC pain & $14 \pm 1.13$ & $10 \pm 0.46$ & $5 \pm 0.51$ & $14 \pm 1.08$ & $11 \pm 1.29$ & $6 \pm 0.57$ \\
\hline WOMAC physical function & $47 \pm 13.01$ & $30 \pm 7.27$ & $15 \pm 1.43$ & $42.5 \pm 13.85$ & $30 \pm 9.91$ & $15 \pm 0.63$ \\
\hline QOL & $63 \pm 3.71$ & $77 \pm 4.06$ & $92 \pm 4.07$ & $63.5 \pm 4.43$ & $75 \pm 3.28$ & $94 \pm 4.02$ \\
\hline STAIX-1 & $25 \pm 3.04$ & $42 \pm .16$ & $62 \pm 5.43$ & $24 \pm 2.97$ & $38 \pm 4.97$ & $54 \pm 5.72$ \\
\hline
\end{tabular}

\section{Discussions}

The objectives of the treatment for the patients diagnosed with knee osteoarthritis were mainly: the reduction of pain, anxiety caused by the COVID-19 pandemic, increased muscle strength, muscle toning, coordination to achieve static and dynamic balance, increased mobility of the joints, quality of life and rapid reintegration into the socio-familial environment.

The applied electrotherapy had an analgesic, antiinflammatory, resorbive purpose. Kinetotherapy was applied in order to improve physical and mental function, whereas the inhibiting role of nociception is known at the local and central level.

The two studied groups of patients were homogeneous in terms of weight, gender and age. The evaluation of patients based on scales enabled the registration of statistically significant values, the value of $p<0.05$, which explains the validation of the working hypothesis.

Table 4. The values of the t-student test in the two groups of patients (T1-T2)

\begin{tabular}{|l|c|c|}
\hline SCALE & L1 GROUP & L2 GROUP \\
\hline VAS & 0.0247 & 0.0258 \\
\hline WOMAC pain & 0.0156 & 0.0086 \\
\hline WOMAC physical function & 0.0435 & 0.0326 \\
\hline QOL & 0.0055 & 0.0038 \\
\hline STAI X-1 & 0.0377 & 0.0311 \\
\hline
\end{tabular}

The L1 group that received analgesic electrotherapy recorded statistically significant values, as it is seen in the literature of specialty. The application of the kinetotherapy program also had a role in reducing the pain phenomenon. On the other hand, the results obtained by reducing the value of the body mass index by doing physical exercises correspond to the data in the literature that explain the role of physical activities in reducing pain and reducing body mass index (7). 
The pain phenomenon in the context of knee osteoarthritis recorded a more significant decrease in the L1 group, which enabled the increase of patients' quality of life and the reduction of the anxious state caused by the disease and the pandemic context. The sensation of pain is closely related to the anxiety level, an observation that is also found in the study of Zubieta who showed that pain is a subjective experience having a genetic predisposition and being partially influenced by the administration of analgesics (20).

\section{Conclusions}

The individualized recovery treatment associated to the kinetotherapeutic program and an adequate diet enabled the attainment of statistically significant results. The analgesic electrotherapy significantly reduced the pain syndrome of the patients for whom it was used. It has been shown that patients' anxiety can influence the pain phenomenon.

Given the conditions caused by the Covid-19 pandemic, the anxiety of the patients who were in the outpatient department to receive recovery treatment was increased. During the treatment, the anxiety condition decreased while the level of pain decreased.

\section{Acknowledgements}

All authors have read and approved this publication and had equal scientific contribution in publishing this material.

\section{Abreviations}

NSAID -The non-steroidal anti-inflammatory drugs

VAS scale - Visual Analog Scale for Pain

WOMAC scale - Western Ontario and McMaster

Universities Osteoarthritis Index

QOL scale -Quality of life

STAI -State Trait Anxiety Inventory

BMI-Body mass index

\section{References:}

1. Naal FD, Impellizzeri FM: How active are patients undergoing total joint arthroplasty?: A systematic review.Clin Orthop Relat Res, 2010, 468: 1891-1904.

2. Stewart $\mathrm{C}$, Leveille SG, Shmerling $\mathrm{RH}$, et al. Management of Persistent Pain in Older Adults: The MOBILIZE Boston Study. J Am Geriatr Soc. 2012;60(11):2081-2086.

3. Blyth FM, Rochat S, Cumming RG, Waite LM, et al. Pain, frailty and comorbidity on older men: the CHAMP Study. Pain. 2008;140(1):224-230.

4. Covinsky KE, Lindquist K, et al. Pain, Functional Limitations, and Aging, J Am Geriatr Soc. 2009 Sep; 57(9): 1556-1561.
5. Chan KK, Chan LW. A qualitative study on patients with knee osteoarthritis to evaluate the influence of different pain patterns on patients' quality of life and to find out patients' interpretation and coping strategies for the disease. Rheumatol Rep, 2011, 3: 9-15.

6. Hootman JM, Helmick CG. Projections of US prevalence of arthritis and associated activity limitations. Arthritis Rheum, 2006, 54: 226-229.

7. Murphy L, Schwartz TA, Helmick CG,et al. Lifetime risk of symptomatic knee osteoarthritis. Arthritis Rheum.2008;59:1207-13.

8. Tuhina Neogi, The Epidemiology and Impact of Pain in Osteoarthritis, Osteoarthritis Cartilage. 2013 Sep; 21(9): 1145-1153.

9. Cazzola M, Atzeni F, Boccassini L, et al. Physiopathology of pain in rheumatology, Reumatismo. 2014 Jun 6;66(1):4-13.

10. Hosu CD, Moisoiu V, Stefancu A, et al. Raman spectroscopy applications in rheumatology, Lasers in Medical Science (2019) 34:827-834.

11. Wijma AJ, van Wilgen CP, Meeus M, Nijs J. Clinical biopsychosocial physiotherapy assessment of patients with chronic pain: The first step in pain neuroscience education, Physiother Theory Pract. 2016 Jul;32(5):36884.

12. Birlutiu V, Stef L, Mitariu SIC, Filip CR, et al. The Biochemical Biomarkers Determination in Alzheimer Dementia. Rev.CHIM.(Bucharest).2018.69(11), 4055 4059.

13. Reid MC, Papaleontiou M, Ong A, et al. Selfmanagement strategies to reduce pain and improve function among older adults in community settings: A review of the evidence. Pain Med. 2008;9:409-424.

14. Abdel-Aziem AA, Soliman ES, et al, Effect of a physiotherapy rehabilitation program on knee osteoarthritis in patients with different pain intensities, J Phys Ther Sci. 2018 Feb; 30(2): 307-312.

15. Dworkin RH, Peirce-Sandner S, Turk DC, et al. Outcome measures in placebo-controlled trials of osteoarthritis: responsiveness to treatment effects in the REPORT database. Osteoarthritis Cartilage.2011;19:483-92.

16. Imamura $\mathrm{M}$, Imamura ST, Kaziyama $\mathrm{HH}$, et al. Impact of nervous system hyperalgesia on pain, disability, and quality of life in patients with knee osteoarthritis: a controlled analysis. Arthritis Rheum. 2008;59:1424-31.

17. Radulescu A, Electroterapie, Editura Medicala S.A. Bucuresti, 2014

18. Silisteanu SC, Antonescu E, Totan M. Study on the importance of medical treatment and physical methods in recovering patients with knee osteoarthritis, Balneo Research Journal, Vol.10, No.2, May 2019 p: 90-97.

19. Silisteanu SC, Mitariu L, Ranga R,et al. Potentiating the Effect of Treatment with Voltaren Gel Using Ultrasonic Frequencies of $1 \mathrm{MHz}$. Rev.CHIM.(Bucharest). 2018;69(7)

20. Zubieta JK, Smith YR, Bueller JA, et al. Regional mu opioid receptor regulation of sensory and affective dimensions of pain. Science. 2001;293:311-5 